\title{
Electrical Control of Quantum Emitters in a Van der Waals Heterostructure
}

Simon J. U. White, ${ }^{1, \#}$, Tieshan Yang ${ }^{1,2, \#}$, Nikolai Dontschuk ${ }^{3}$, Chi Li ${ }^{1}$, Zai-Quan Xu${ }^{1}$, Mehran Kianinia $^{1,2}$, Alastair Stacey ${ }^{4}$, Milos Toth ${ }^{1,2}$ and Igor Aharonovich ${ }^{1,2}$

1. School of Mathematical and Physical Sciences, University of Technology Sydney, Ultimo, New South Wales 2007, Australia

2. ARC Centre of Excellence for Transformative Meta-Optical Systems, University of Technology Sydney, Ultimo, New South Wales 2007, Australia

3. School of Physics, University of Melbourne, Parkville, Victoria 3010, Australia

4. School of Science, RMIT University, Melbourne, Victoria 3001, Australia

\# These authors contributed equally

Controlling and manipulating individual quantum systems in solids underpins the growing interest in development of scalable quantum technologies. Recently, hexagonal boron nitride $(\mathrm{hBN})$ has garnered significant attention in quantum photonic applications due to its ability to host optically stable quantum emitters. However, the large band gap of $h B N$ and the lack of efficient doping inhibits electrical triggering and limits opportunities to study electrical control of emitters. Here, we show an approach to electrically modulate quantum emitters in $n h B N-$ graphene van der Waals heterostructure. We show that quantum emitters in $h B N$ can be reversibly activated and modulated by applying a bias across the device. Notably, a significant number of quantum emitters are intrinsically dark, and become optically active at non-zero voltages. To explain the results, we provide a heuristic electrostatic model of this unique behaviour. Finally, employing these devices we demonstrate a nearly-coherent source with linewidths of $\sim 160 \mathrm{MHz}$. Our results enhance the potential of hBN for tuneable solid state quantum emitters for the growing field of quantum information science.

Van der Waals (vdW) heterostructures have emerged as a fascinating platform to study lightmatter interaction at the nanoscale ${ }^{1-11}$. Assembling various atomically thin crystals has enabled the observation of new physical phenomena in these unconventional materials, including superconductivity $^{12}$, interlayer excitons ${ }^{13}$, moire lattices ${ }^{3,14}$ and correlated electronic systems ${ }^{15}$. Furthermore, advanced practical devices such as broadband photodetectors, efficient light emitting diodes and nanoscale lasers have also been realized from a variety of vdW crystals ${ }^{16}$. Indeed, control over light emission from a selected family of transition metal di-chalcogenides enabled optical detection of valley states, and observation of exciton-polariton condensates even at room temperature ${ }^{17-20}$.

Of particular interest is the ability to manipulate light emission from single point defects, commonly referred to as single photon emitters (SPEs), as they are critical building blocks for quantum technologies ${ }^{1}$. Hexagonal boron nitride (hBN), a wide band gap vdW crystal, has been extensively studied in recent years as a vdW host of SPEs that are ultra bright and optically stable. In addition, hBN SPEs exhibit spin - photon interface and can be engineered on demand in an atomically thin crystal ${ }^{21,22}$. This combination of photophysical properties foreshadows ample opportunities for their utilisation as quantum sources and quantum repeaters in scalable quantum photonic devices. An outstanding challenge for solid state SPEs is to realize electrical 
control of the optical emission. This challenge stems from the fact that most hosts of defectbased SPEs are wide band gap materials in which p-type or n-type doping is limited ${ }^{23,24}$. Indeed, even for well-studied materials such as diamond or silicon carbide, electrical modulation of quantum emitters is limited to specific defects and often requires cumbersome device engineering ${ }^{25-28}$.

Here we demonstrate a facile and scalable approach to electrically modulate quantum emitters in $\mathrm{hBN}$ - graphene heterostructures. Our experiments show that SPEs in hBN can be controllably activated and modulated by applying a voltage across the devices. Intriguingly, we show that most of the quantum emitters become optically active at non-zero voltages, in contrast to what has been observed in the case of defects in 3D crystals. We interpret our results in the context of electrically-induced changes in the charge states of the hBN defects and provide electrostatic models to support the experimental findings.

Figure $1 \mathrm{a}$ is a schematic illustration of the heterostructure devices used in this study. The device structure consists of multilayer graphene (MLG), a hBN capping layer and a hBN emitter layer stacked vertically on p-type silicon with a $285 \mathrm{~nm}$ thermal oxide. Bias is applied between the bottom p-type silicon and MLG. An optical image of the device is shown in Figure 1b. The black, light blue and green dashed lines indicate the boundaries of the MLG, the hBN capping layer and the hBN layer that hosts the quantum emitters, respectively. The capping layer $(\sim 20$ $\mathrm{nm}$ ) is used to prevent quenching of emitters in the active hBN layer by MLG. Details of the fabrication process can be found in the methods section.
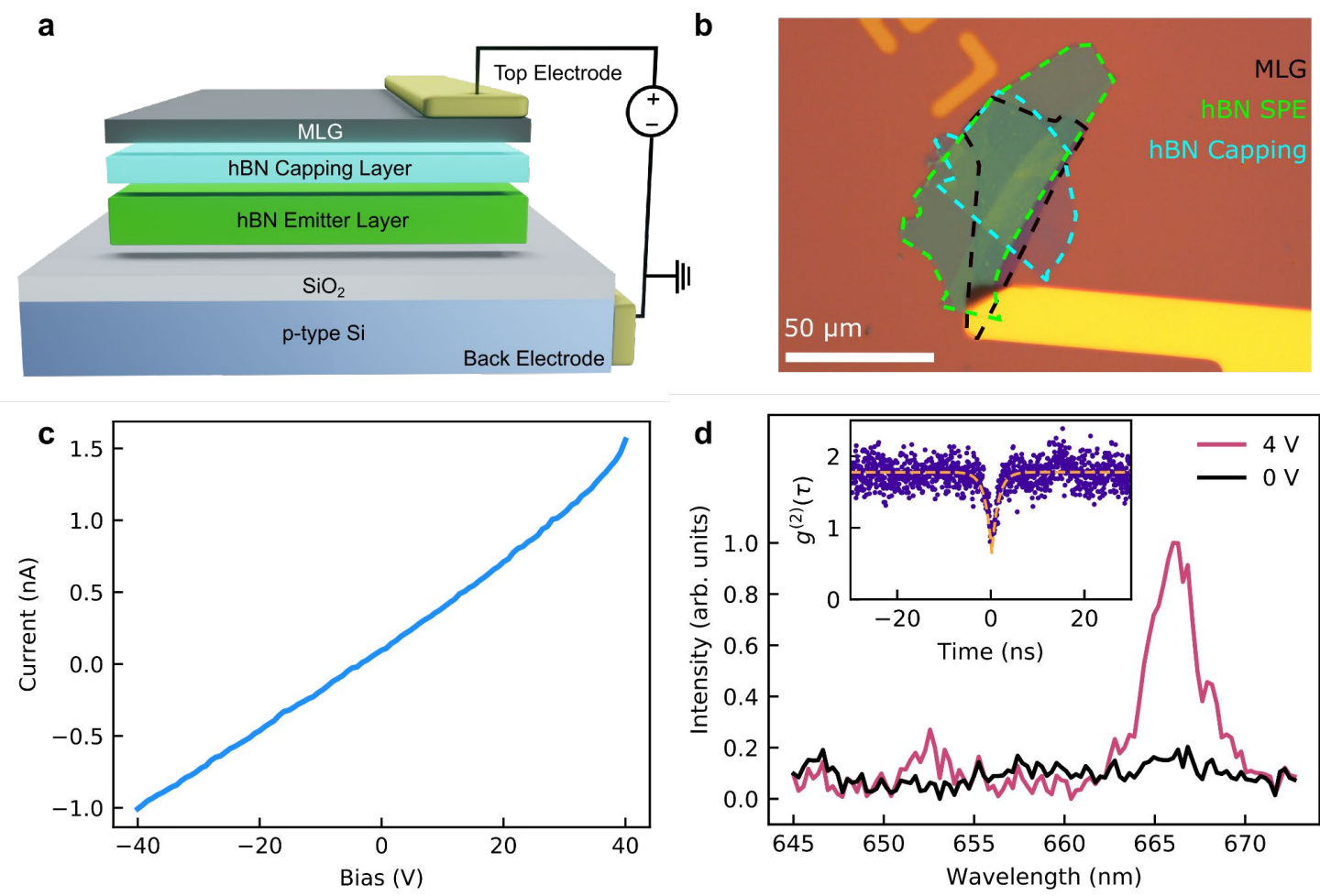

Figure 1: The hBN/MLG heterostructure device. a. Schematic of the device and its operating principle. The device is biased using gold electrodes in contact with $M L G$ and p-type silicon. $\boldsymbol{b}$. Optical image of a device consisting of MLG, a hBN capping layer and a hBN layer that contains SPEs. The substrate is p-type silicon with a $285 \mathrm{~nm}$ thermal oxide layer. Each layer is outlined by dashed lines. $\boldsymbol{c}$. I-V cure from the device showing a negligible leakage current. $\boldsymbol{d}$. Normalized PL spectra of an emitter 
in the heterostructure at a bias of 0 (black) and $4 V$ (purple), respectively. Inset: second autocorrelation function measured from the emitter at $4 \mathrm{~V}$, confirming its a single photon source.

To characterise the device, we first measured a current-voltage (I-V) curve by sweeping the bias from $-40 \mathrm{~V}$ to $40 \mathrm{~V}$. The current scales linearly with voltage, as shown in Figure $3 \mathrm{c}$, and the maximum measured current is less than $1.5 \mathrm{nA}$. This is an upper bound on the current through the hBN layers since the top electrode is in contact with both the MLG and the oxide layer (see Figure 1b). The I-V curve shows that the device behaves as a capacitor that generates an electric field within the $\mathrm{hBN}$ layers. Additional electrical measurements from the device are included in the supporting information.

Next, we study the optical properties of quantum emitters embedded in the heterostructure. All optical measurements were performed using a $532 \mathrm{~nm}$ continuous wave excitation laser, and a custom-built confocal microscope (see methods for details). Figure 1d shows photoluminescence (PL) spectra from one emitter at ambient conditions, using a bias of 0 (black curve) and $4 \mathrm{~V}$ (purple curve). Remarkably, a clear peak at $666 \mathrm{~nm}$ arises when the voltage is switched from 0 to $4 \mathrm{~V}$, indicating activation of the emitter by the applied bias. A second-order autocorrelation function measured from this emitter is shown in the inset of Figure 1d. The dip at zero delay time confirms that the defect is a single photon emitter. Additional spectra of emitters are presented in the SI.

To elucidate this peculiar behaviour, the hBN/MLG heterostructure device was loaded into a closed cycle He cryostat operating at $4 \mathrm{~K}$. Electrical control of the hBN quantum emitters is shown in Figure 2. The bias-dependence of PL spectra from two emitters are plotted in Figure $2 \mathrm{a}$ and $2 \mathrm{~b}$. The spectra are normalised for clarity, and illustrate two distinct behaviours observed predominantly at under positive (Figure 2a) and negative (Figure 2b) bias applied to the MLG electrode. The emitter in Figure 2a does not fluoresce at zero bias. However, as the bias is increased, the emitter becomes active at $\sim 8 \mathrm{~V}$, the brightness increases up to $\sim 15 \mathrm{~V}$ where it goes through a maximum and decreases as the bias is increased further. It becomes inactive at $\sim 22 \mathrm{~V}$, and is not restored within our experimental conditions. On the other hand, the emitter in Figure $2 \mathrm{~b}$ shows a completely different behavior. As the bias is reduced from $0 \mathrm{~V}$ to $-30 \mathrm{~V}$, the emission intensity increases gradually, and remains optically active even under $30 \mathrm{~V}$. This is unexpected, given that under positive biases, there was only a window of voltages under which the emission was persistent. This would be explained later in detail. Note, that in both cases, a minor shift of the emission was observed, as expected, due to the stark shift. The direction of the Stark shifts depends on the polarity of the applied bias and the dipole orientation of each emitter.

The emission intensity can be further tuned dynamically with the applied bias. This is shown in Figure 2c, where an emitter is modulated using a square wave voltage function oscillating between 0 and $+10 \mathrm{~V}$. The period of the intensity resembles the square wave bias function, illustrating repeatability of the activation process - the switching is reversible and repeatable, and no significant blinking or bleaching of the emitter occurs during the measurement. Similar behavior was also observed for emitters activated by a negative bias applied to the MLG. 

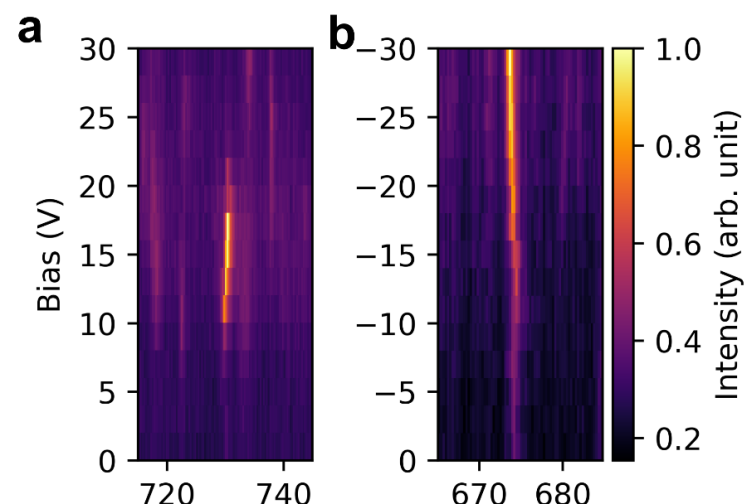

Wavelength $(\mathrm{nm})$ Wavelength $(\mathrm{nm})$

d

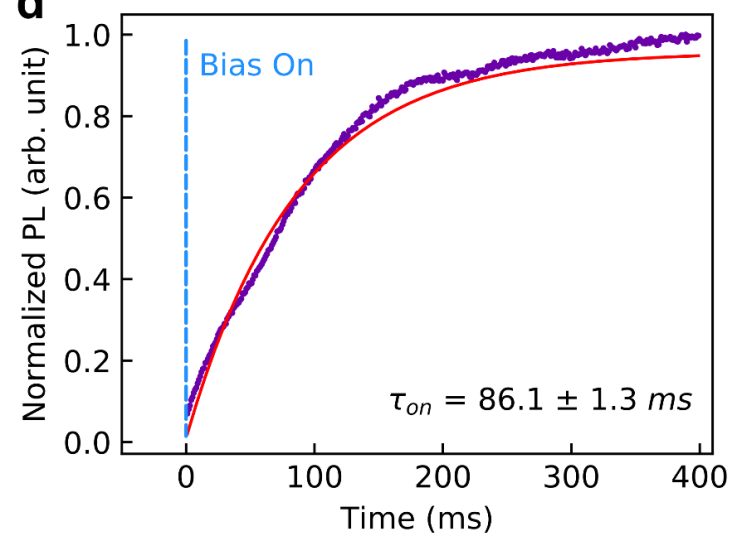

f

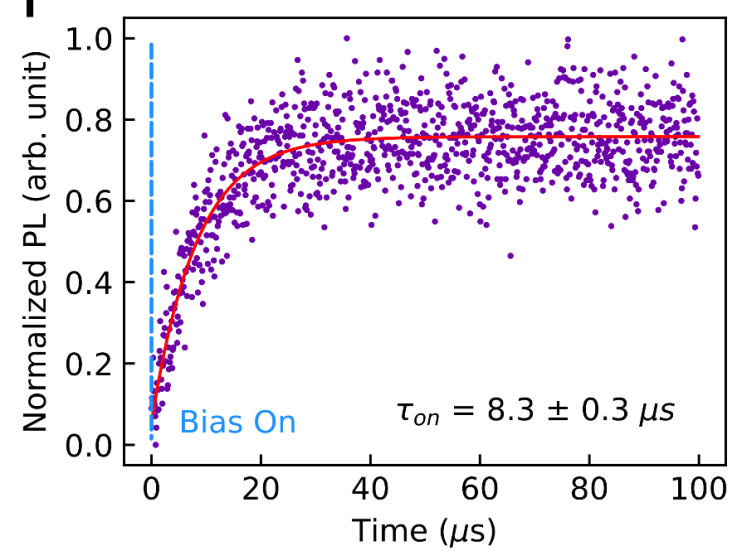

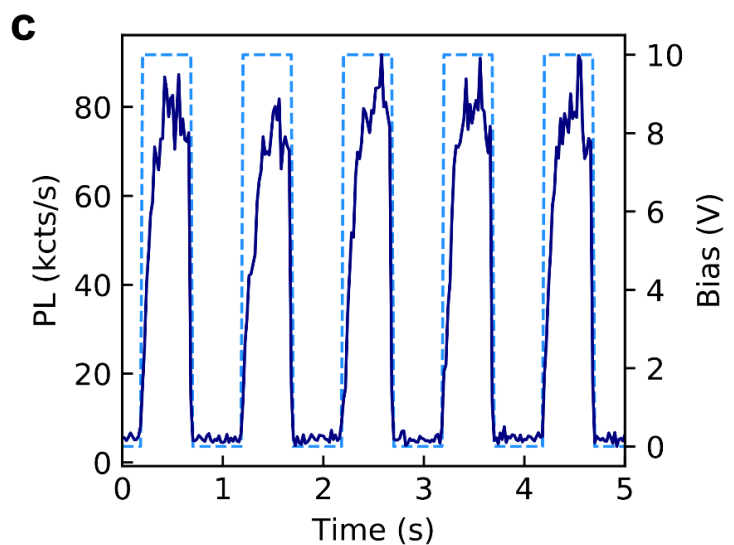

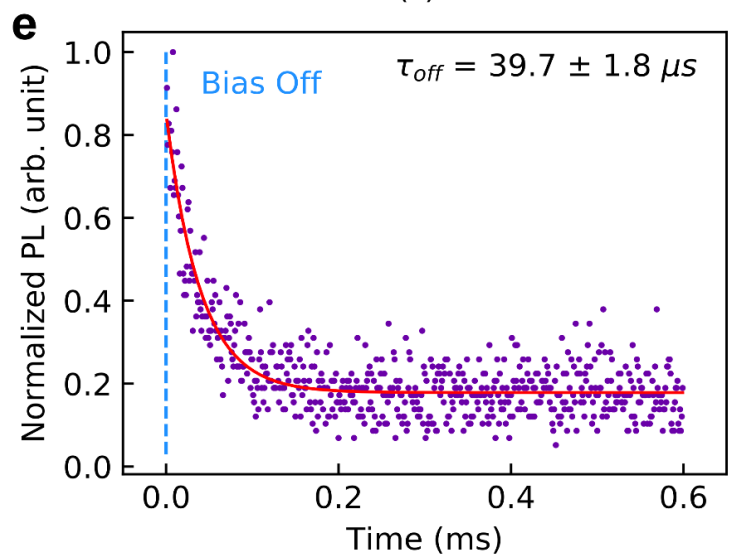

g

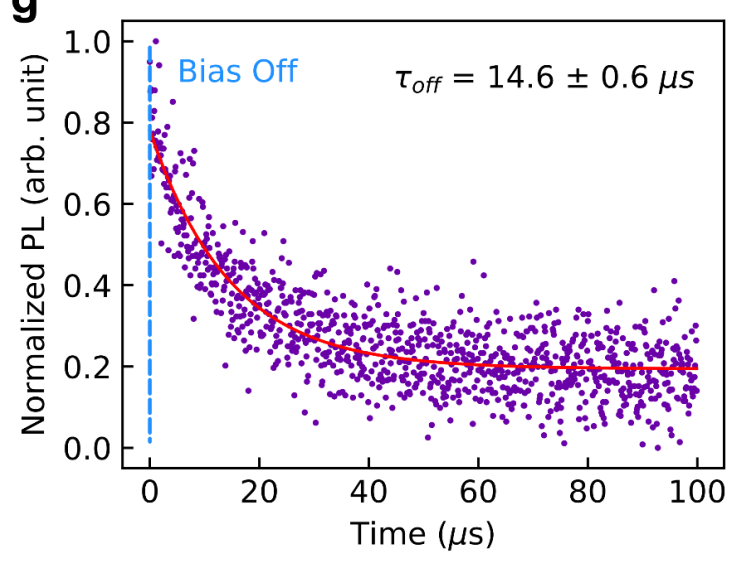

Figure 2: Electrical control of hBN quantum emitters in the heterostructure device. a, b. Normalized $P L$ spectra recorded from two different emitters as the bias applied to MLG was varied from $0 \mathrm{~V}$ to +30 $V(a)$, and $-30 V(b)$. c. Dynamic modulation of the emission intensity of a quantum emitter by a square wave bias function. The bias is switched periodically between $0 \mathrm{~V}$ and $+10 \mathrm{~V}$, as shown by the light blue trace. The filtered PL signal intensity detected by an avalanche photodiode (APD) is plotted in dark blue. $\boldsymbol{d}, \boldsymbol{e}$. Normalised PL intensity versus time, showing the emission dynamics when the emitter is turned on (d) and off (e) by a $+10 \mathrm{~V}$ step function applied to the MLG electrode. The measured data are fitted with single exponential functions, and the time constants, $\tau_{o n}$ and $\tau_{o f f}$, are $86 \mathrm{~ms}$ and $40 \mu \mathrm{s}$, respectively. $\boldsymbol{f}$, g. Corresponding dynamics from an emitter that becomes active under negative bias, measured by applying a $-10 \mathrm{~V}$ step function to the MLG electrode. Under negative bias $\tau_{\text {on }}$ and $\tau_{\text {off }}$ are $8 \mu$ s and $15 \mu$ s, respectively.

A detailed analysis of the switching rates is presented in Figure 2d-g. The time-correlated intensity was recorded using a time tagger (Swabian instrument, jitter of $<200 \mathrm{ps}$ ) whilst bias 
step functions were applied to the device. Figure 2d,e show the PL rise and decay times when a bias of $+10 \mathrm{~V}$ was turned on and off, respectively. The curves were fitted with single exponential functions and the rise $\left(\tau_{\text {on }}\right)$ and fall times $\left(\tau_{\text {off }}\right)$ are estimated to be $\sim 86 \mathrm{~ms}$ and $\sim$ $40 \mu$ s, respectively. The rise time is $\sim 2000$ times slower than the fall time, indicating significant differences between the charging and discharging dynamics ${ }^{29}$.

The corresponding measurements obtained using a negative bias of $-10 \mathrm{~V}$ are shown in Figure $2 \mathrm{f}, \mathrm{g}$. Under negative bias, $\tau_{\text {on }}$ and $\tau_{\text {off }}$ are comparable, approximately $8 \mu$ s and $15 \mu$ s, respectively. Strikingly, the rise time under negative bias is over four orders of magnitude faster than under the negative bias, whilst the fall times are similar under both positive and negative bias. The dramatic difference between the rise times is indicative of distinct emitter activation mechanisms under positive and negative bias, as is discussed in detail below.

To provide a broad, statistically-representative overview of the behaviour of quantum emitters under applied bias, we recorded PL spectra from a large ensemble of quantum emitters within the area of a single excitation laser spot. The spectra recorded as a function of bias over the range of $-40 \mathrm{~V}$ to $+40 \mathrm{~V}$ is shown in Figure $3 \mathrm{a}$, where each emission line corresponds to a single quantum emitter in hBN. The lines at $580 \mathrm{~nm}(620 \mathrm{~nm})$ are the $\mathrm{G}(2 \mathrm{D})$ bands of MLG and remain unchanged (at this particular spectrometer resolution).

A large number of emitters spanning a broad range of emission wavelengths are activated when a positive bias is applied to the MLG electrode, mostly above $+10 \mathrm{~V}$. Similarly, numerous emission lines appear when a negative bias is applied to the device, and become increasingly brighter as the bias decreases to $-40 \mathrm{~V}$. We note that no emission was observed from the device at any bias in the absence of the excitation laser - that is, all emissions discussed in this paper are field-activated PL rather than electroluminescence.

To investigate this effect further, we plot the intensity from a number of representative emitters as a function of applied bias in Figure $3 b$ and figure $3 c$. Figure $3 b$ shows four emissions that are active within a positive bias range. The PL intensity from each of these emitters is highly bias-dependent. For example the intensity of the $581 \mathrm{~nm}$ line peaks at a bias of $\sim 10 \mathrm{~V}$, while the $641 \mathrm{~nm}$ line peaks at $\sim 28 \mathrm{~V}$. Interestingly, most of the emitters have a clear bias activation range - that is, they are optically active over this range and inactive at biases outside this range. Such behaviour has never been observed for any other solid-state quantum emitters, and it is discussed in detail below.

The behaviour is substantially different when a negative bias is applied to the MLG electrode. As is shown in Figure 3a, as the bias is reduced from $0 \mathrm{~V}$ to $-40 \mathrm{~V}$, a number of emitters become optically active and none of them deactivate over the entire bias range. The intensity of a number of representative emissions from this group is plotted versus bias in Figure $3 \mathrm{c}$. The emitters are very dim at zero bias, and the emission intensities increase linearly as the bias is reduced from 0 to $-40 \mathrm{~V}$ under constant laser excitation power. We note that an increase in emitter intensity versus bias has been observed previously for neutrally charged NV centres in diamond $^{25,30}$. However, more broadly, the observation of PL emissions that are inactive until a voltage is applied has not been reported for any solid state quantum systems. 

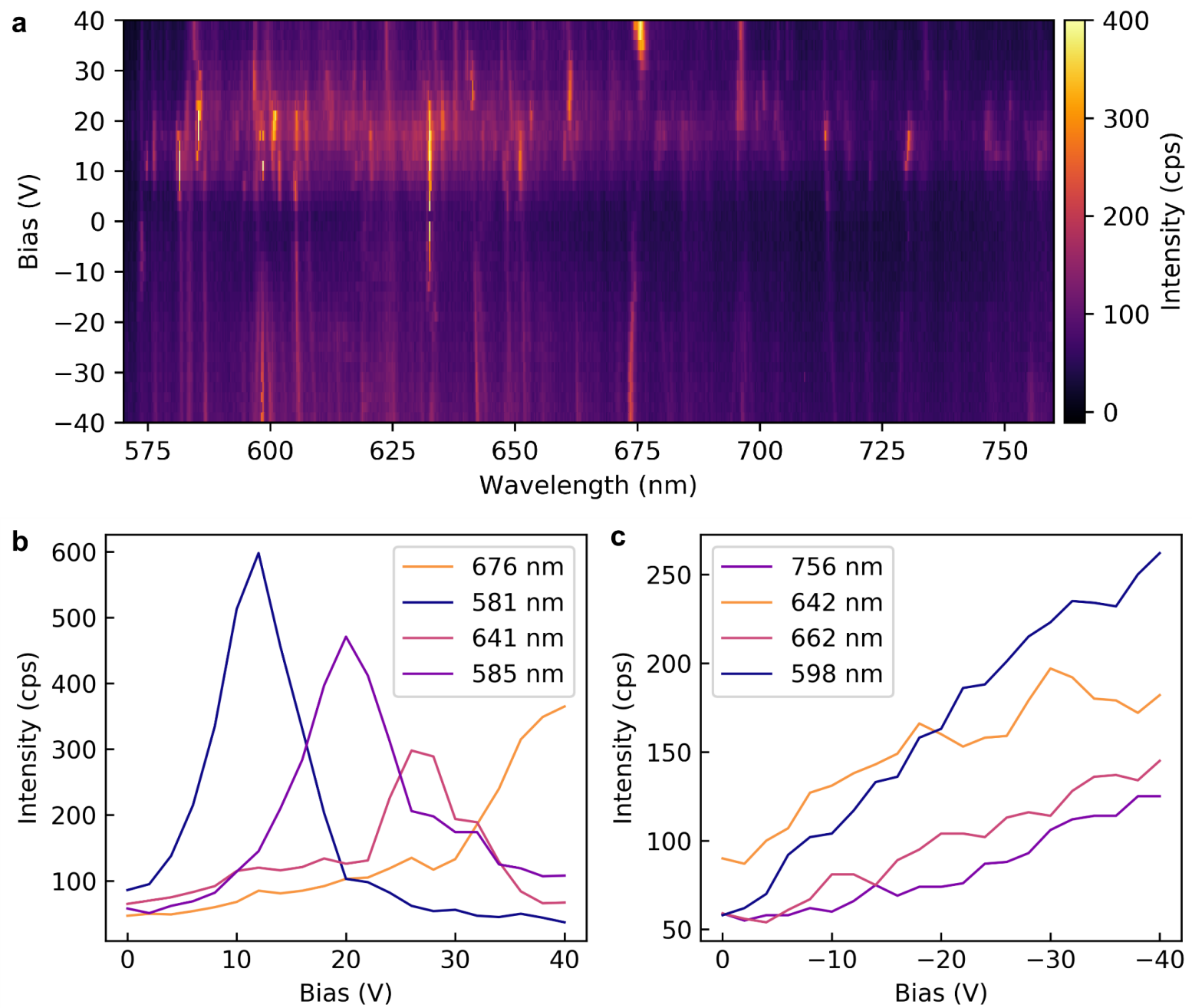

Figure 3: Activation of hBN quantum emitters in the heterostructure device. a. PL spectra recorded as a function of bias, over the range of -40 to $+40 \mathrm{~V}$. The lines at $580 \mathrm{~nm}$ and $620 \mathrm{~nm}$ are the $G$ band and $2 D$ band of $M L G$. The remaining lines are quantum emitters in $h B N$. $\boldsymbol{b}, \boldsymbol{c}$ Emission intensity versus bias for a number of emitters activated by a positive (b) and a negative (c) voltage applied to the MLG electrode.

We now turn to a discussion of the photophysics of these emitters under applied bias. We attribute the emitter activation and deactivation caused by a positive bias (seen in Figure $3 b$ ) to changes in charge states of defects in $\mathrm{hBN}$, and the activation of emitters under negative bias (seen in Figure 3c) to the injection of hot electrons from MLG into hBN. These two processes are characterised by the slow and fast emitter activation dynamics, as is discussed below in the context of the electron energy level diagram shown in Figure 4.

The device band diagram under zero bias is shown in Figure 4a. The MLG split Fermi level, $\mathrm{E}_{\mathrm{F}}$, and the bottom of the hBN conduction band are located 4.6 and $2.3 \mathrm{eV}$ below the vacuum level, respectively. Also shown on the diagram are two hypothetical charge transition levels of a defect in hBN, adapted from reported density functional theory (DFT) calculations ${ }^{31,32}$. Figures $4 \mathrm{~b}$ and $\mathrm{c}$ show the device at a bias of +10 and $+20 \mathrm{~V}$, respectively, and illustrate how a positive bias sweep causes sloping of the energy bands, and an effective sweep of $E_{F}$ within a subset of the band gap of hBN. A defect with a charge transition level within this region of the band gap will gain/lose an electron as $E_{F}$ moves above/below the level (Figure $4 \mathrm{~b}$ ). 
Similarly, a defect with two charge transition levels in this region of the band gap will change charge state twice if $\mathrm{E}_{\mathrm{F}}$ sweeps through both levels. Hence, the hBN defect in Figure 4a will have lost two electrons upon the application of $+20 \mathrm{~V}$ to the MLG (Figure 4c). Each change in the charge state of an emitter will result in a corresponding change in the defect energy levels and hence the emission spectrum ${ }^{25,30}$. Importantly, a change in charge state often causes activation or deactivation of an emitter - either absolutely or effectively by causing the emission energy to shift outside the measured spectral range ${ }^{25}$. Hence, activation of a hBN quantum emitter upon the application of a positive bias to the MLG electrode of our heterostructure device can be caused by a change in the charge state of the emitter by +1 (Figure $4 b)$. Deactivation of the emitter at a greater positive bias can be caused by a second change in charge state, provided that $\mathrm{E}_{\mathrm{F}}$ crosses a second charge transition level of the emitter (Figure 4c). For completeness, we should point out that the voltage sweeps also affect defect states that are in the vicinity of and can modulate quantum emitters in hBN.

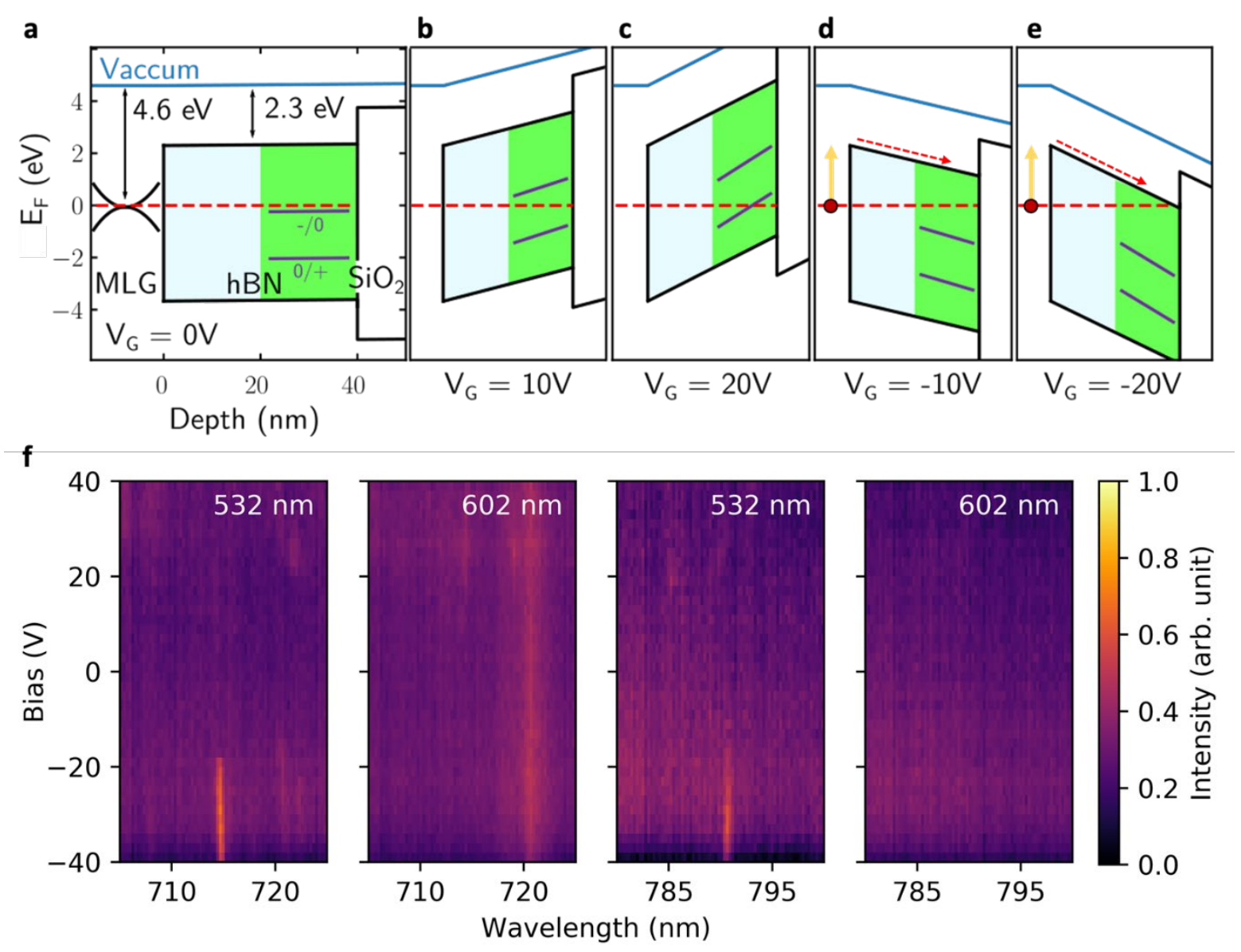

Figure 4: Band diagram of the heterostructure device under various bias configurations. a. The device with both electrodes grounded $\left(V_{G}=0 V\right)$. The $h B N$ capping layer is shown in light blue and the $h B N$ layer that contains quantum emitters is shown in green (the thickness of each $h B N$ layer is assumed to be $20 \mathrm{~nm}$ ). The $M L G$ split fermi level $\left(E_{F}\right)$ extends into the $h B N$, indicating charge transfer between $M L G$ and defect states in $h B N$ (see text). The purple lines indicate two hypothetical charge transition levels of a single defect in $h B N$. b,c. The device with a bias of $+10 \mathrm{~V}(\mathrm{~b})$ and $+20 \mathrm{~V}$ (c) applied to the MLG electrode. d,e. The device with a bias of $-10 \mathrm{~V}(\mathrm{~d})$ and $-20 \mathrm{~V}$ (e) applied to the MLG electrode. The solid yellow arrows show 
photoexcitation of an electron in MLG, and broken red arrows indicate drift of the electrons via the band gaps of the $h B N$ layers. $f$. Experimental verification of the model, whereby emitters are only visible under green excitation (at $715 \mathrm{~nm}$ and $790 \mathrm{~nm}$ ), but not under lower energy red excitation, at the same confocal spot. The emission at $720 \mathrm{~nm}$ is the graphene Raman.

Based on the above, activation of an emitter upon application of a negative bias could be argued to be caused by a change in the charge state of the emitter by -1 . However, an upward sweep of $\mathrm{E}_{\mathrm{F}}$ within the bandgap of $\mathrm{hBN}$ will populate deep defect levels and we do not expect it to activate emitters. Moreover, we found that the activation rate measured by applying a step voltage function to the device is over three orders of magnitude slower for the case of positive bias than for the case of negative bias (Figure $2 \mathrm{~d}$ and $\mathrm{f}$, respectively), indicating a fundamental difference in the charge transfer dynamics. To explain this difference, we consider energy band diagrams for the negatively charged device shown in Figure $4 \mathrm{~d}$ and e for the case of -10 and $20 \mathrm{~V}$, respectively. Application of a bias that is negative with respect to the MGL electrode inverts the gradient of the sloped bands and effectively raises $\mathrm{E}_{\mathrm{F}}$ towards the $\mathrm{hBN}$ conduction band. In this configuration, electrons excited in the MLG by the laser (yellow arrows in Figure 4) can tunnel across the barrier at the MLG-hBN interface and drift (red broken arrows) within $\mathrm{hBN}$ under the influence of the applied electric field. The resulting photocurrent provides a means to supply hot electrons to emitters via the hBN conduction band. This charge transfer mechanism is therefore expected to be fast relative to the case of a positive bias (Figure 4b,c), where electron removal from the deep hBN charge transition levels likely occurs via a hopping mechanism and electrons flow to the MLG via trap states inside the hBN band gap.

The above analysis illustrates two distinct charge transfer mechanisms between the MLG electrode and defects in hBN, which are slow/fast in the case of positive/negative bias applied to the MLG. The first can account for emitter activation and deactivation upon application of a positive voltage sweep to the device, and the second can account for emitter activation by a negative bias. We note that the almost universal deactivation of emitters at $+40 \mathrm{~V}$ seen in Figure 4a is likely a consequence of the fact that $\mathrm{E}_{\mathrm{F}}$ lies very close to the $\mathrm{hBN}$ valence band and the ground states of most emitters are ionised at this voltage. We also note that, as is evident from Figure 4, the voltage needed to activate/deactivate various emitters is a function of the emitter location within the hBN. This observation combined with the fact that a number of distinct defect species are responsible for the rich emission spectrum of $\mathrm{hBN}$ accounts for the variation in activation and deactivation voltages seen in Figure $3 \mathrm{a}$.

To provide further experimental support for our model, we increased the excitation laser wavelength from $532 \mathrm{~nm}(\sim 2.3 \mathrm{eV})$ to $602 \mathrm{~nm}(\sim 2 \mathrm{eV})$. The longer wavelength excitation should not be sufficient to overcome the energy barrier (see figure $4 \mathrm{~d}$,e) under negative bias, and hence no emitters should be activated. Indeed, this hypothesis is confirmed. Figure $4 \mathrm{f}$ shows PL spectra of emitters under positive and negative bias recorded at the same confocal spot using the two excitation wavelengths. It is clear that new emitters appear under negative bias when a $532 \mathrm{~nm}$ excitation laser is used, but no emission appears under the longer excitation wavelength of $602 \mathrm{~nm}$.

To illustrate the potential of our devices for practical and scalable quantum photonic applications, we demonstrate resonant excitation of these quantum emitters under a negative bias. We expect that under these conditions, the electric field and photocurrent will govern the 
charge states of both emitters and surrounding charge traps and thus suppress charge fluctuations and spectral diffusion. Furthermore, the photocurrent is expected to re-activate emitters after each cycle, without the need for a repumping laser, as is common for most other solid state defects, including defects in hBN. This was indeed observed, as is illustrated in figure 5. Figure 5a shows an emitter with a ZPL at $\sim 588.5 \mathrm{~nm}$, recorded from the device using an off-resonant $532 \mathrm{~nm}$ excitation laser. The off-resonant linewidth is phonon broadened as expected. Figure $5 \mathrm{~b}$ shows a resonant excitation scan of the same emitter with a measured linewidth of $\sim 158 \pm 19 \mathrm{MHz}$. Both measurements were taken using an applied bias, $\mathrm{V}_{\mathrm{G}}=-40 \mathrm{~V}$, and importantly, no resonant emission was observed at zero bias. For quantum emitters in hBN with excited state lifetimes on the order of $\sim 3 \mathrm{~ns}, \sim 160 \mathrm{MHz}$ certainly represents a nearlycoherent, Fourier Transform limited, linewidth, which is highly promising for futuregeneration indistinguishable photons.
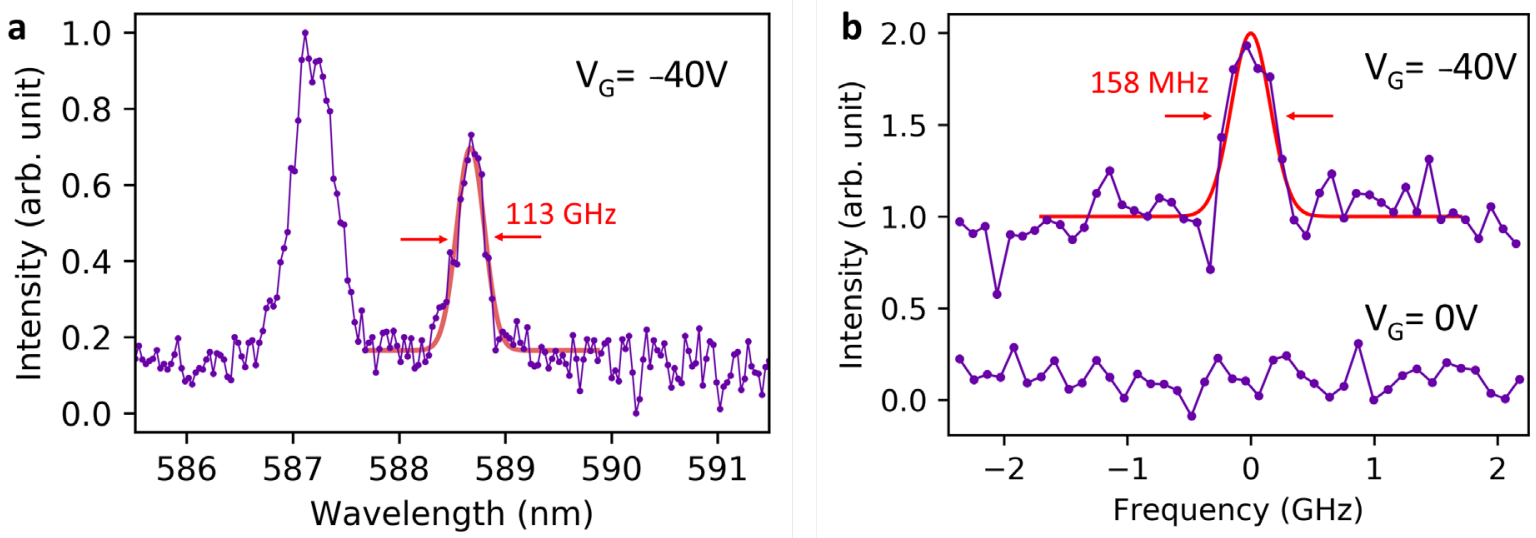

Figure 5. Coherent excitation of quantum emitters in hBN. a. Emission spectrum of a single emitter with a ZPL at $588.5 \mathrm{~nm}$, recorded under non-resonant $532 \mathrm{~nm}$ excitation. b. Resonant excitation of the same emitter, resulting in a nearly-coherent photon source with a linewidth of $\sim 158 \mathrm{MHz}$. Both measurements were done using a bias voltage, $V_{G}=-40 \mathrm{~V}$.

To summarise, we demonstrate electrical modulation and control of a variety of quantum emitters in a vdW heterostructure. The quantum opto-electronic devices consist of MLG/hBN heterostructures, operate at accessible voltages and can be assembled using readily-accessible fabrication techniques. We propose two distinct mechanisms for device operation versus bias polarity based on electrostatic charge switching of quantum emitters and drift of hot photoelectrons. Our results open a plethora of new opportunities in integrated quantum photonics with vdW materials. First, the ability to modulate and switch on/off quantum emitters is imperative for scalable quantum circuitry. Second, electrostatic gating can now be used to activate emitters post $\mathrm{hBN}$ growth and processing, and to select emitters at specific wavelengths. Third, a single device can now be employed to activate and tune emitters into resonance to achieve indistinguishable photons from quantum emitters in hBN. Indeed, our results already show that under negative bias a nearly-coherent quantum source in $\mathrm{hBN}$ with linewidths of $\sim 160 \mathrm{MHz}$ can be obtained. Finally, and equally important, our results constitute the possibility to characterise charge transition levels of specific defects in $\mathrm{hBN}$, and correlate them with theoretical studies of specific atomic defect structures. 


\section{Acknowledgements}

The authors acknowledge financial support from the Australian Research Council (CE200100010, DP190101058) and the Asian Office of Aerospace Research \& Development (FA2386-20-1-4014). The authors thank the Australian Nanofabrication Facilities at the UTS OptoFab node.

\section{Author Contributions}

I.A., Z.X., M.K., and S.W. conceived the idea and designed experiments. T.Y. carried out the device fabrication, C.L., and Z.X. aided in emitter engineering and S.W. carried out the cryogenic measurements. S. W., M. K., T.Y. plotted and analysed the data with input from all coauthors. N.D. and A.S. performed theoretical simulations. I.A. M. T. and M. K. supervised the work and wrote the manuscript with input from all coauthors.

\section{References}

1. Atatüre, M.; Englund, D.; Vamivakas, N.; Lee, S.-Y.; Wrachtrup, J., Material platforms for spin-based photonic quantum technologies. Nature Reviews Materials 2018, 3, 38-51.

2. $\quad$ Elshaari, A. W.; Pernice, W.; Srinivasan, K.; Benson, O.; Zwiller, V., Hybrid integrated quantum photonic circuits. Nat. Photonics 2020, 14 (5), 285-298.

3. McGilly, L. J.; Kerelsky, A.; Finney, N. R.; Shapovalov, K.; Shih, E.-M.; Ghiotto, A.; Zeng, Y.; Moore, S. L.; Wu, W.; Bai, Y.; Watanabe, K.; Taniguchi, T.; Stengel, M.; Zhou, L.; Hone, J.; Zhu, X.; Basov, D. N.; Dean, C.; Dreyer, C. E.; Pasupathy, A. N., Visualization of moiré superlattices. Nature Nanotech. 2020, 15 (7), 580-584.

4. Hwangbo, K.; Zhang, Q.; Jiang, Q.; Wang, Y.; Fonseca, J.; Wang, C.; Diederich, G. M.; Gamelin, D. R.; Xiao, D.; Chu, J.-H.; Yao, W.; Xu, X., Highly anisotropic excitons and multiple phonon bound states in a van der Waals antiferromagnetic insulator. Nature Nanotech. 2021, 16 (6), 655-660.

5. Geim, A. K.; Grigorieva, I. V., Van der Waals heterostructures. Nature 2013, 499 (7459), 419-425.

6. Jiang, Y.; Chen, S.; Zheng, W.; Zheng, B.; Pan, A., Interlayer exciton formation, relaxation, and transport in TMD van der Waals heterostructures. Light: Science \& Applications 2021, 10 (1), 72.

7. Mendelson, N.; Chugh, D.; Reimers, J. R.; Cheng, T. S.; Gottscholl, A.; Long, H.; Mellor, C. J.; Zettl, A.; Dyakonov, V.; Beton, P. H.; Novikov, S. V.; Jagadish, C.; Tan, H. H.; Ford, M. J.; Toth, M.; Bradac, C.; Aharonovich, I., Identifying carbon as the source of visible single-photon emission from hexagonal boron nitride. Nature Mater. 2020.

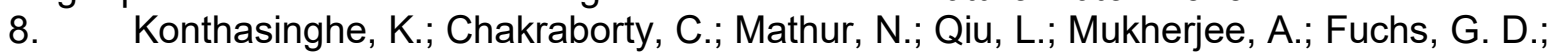
Vamivakas, A. N., Rabi oscillations and resonance fluorescence from a single hexagonal boron nitride quantum emitter. Optica 2019, 6 (5), 542-548.

9. $\quad$ Stewart, J. C.; Fan, Y.; Danial, J. S. H.; Goetz, A.; Prasad, A. S.; Burton, O. J.; Alexander-Webber, J. A.; Lee, S. F.; Skoff, S. M.; Babenko, V.; Hofmann, S., Quantum Emitter Localization in Layer-Engineered Hexagonal Boron Nitride. ACS Nano 2021, 15 (8), 13591-13603.

10. Fournier, C.; Plaud, A.; Roux, S.; Pierret, A.; Rosticher, M.; Watanabe, K.; Taniguchi, T.; Buil, S.; Quélin, X.; Barjon, J.; Hermier, J.-P.; Delteil, A., Position-controlled quantum emitters with reproducible emission wavelength in hexagonal boron nitride. Nat. Commun. 2021, $12(1), 3779$.

11. Vogl, T.; Lecamwasam, R.; Buchler, B. C.; Lu, Y.; Lam, P. K., Compact CavityEnhanced Single-Photon Generation with Hexagonal Boron Nitride. ACS Photonics 2019, 6 (8), 1955-1962. 
12. Cao, Y.; Fatemi, V.; Fang, S.; Watanabe, K.; Taniguchi, T.; Kaxiras, E.; JarilloHerrero, P., Unconventional superconductivity in magic-angle graphene superlattices. Nature 2018, 556, 43.

13. Montblanch, A. R. P.; Kara, D. M.; Paradisanos, I.; Purser, C. M.; Feuer, M. S. G.; Alexeev, E. M.; Stefan, L.; Qin, Y.; Blei, M.; Wang, G.; Cadore, A. R.; Latawiec, P.; Lončar, M.; Tongay, S.; Ferrari, A. C.; Atatüre, M., Confinement of long-lived interlayer excitons in WS2/WSe2 heterostructures. Communications Physics 2021, 4 (1), 119.

14. Baek, H.; Brotons-Gisbert, M.; Koong, Z. X.; Campbell, A.; Rambach, M.; Watanabe, K.; Taniguchi, T.; Gerardot, B. D., Highly energy-tunable quantum light from moir\&\#xe9;trapped excitons. 2020, 6 (37), eaba8526.

15. Wang, L.; Shih, E.-M.; Ghiotto, A.; Xian, L.; Rhodes, D. A.; Tan, C.; Claassen, M.; Kennes, D. M.; Bai, Y.; Kim, B.; Watanabe, K.; Taniguchi, T.; Zhu, X.; Hone, J.; Rubio, A.; Pasupathy, A. N.; Dean, C. R., Correlated electronic phases in twisted bilayer transition metal dichalcogenides. Nature Mater. 2020, 19 (8), 861-866.

16. Fu, J.; Quu, M.; Bao, W.; Zhang, H., Frontiers in Electronic and Optoelectronic Devices Based on 2D Materials. Advanced Electronic Materials 2021, 7 (7), 2100444.

17. Lundt, N.; Klembt, S.; Cherotchenko, E.; Betzold, S.; Iff, O.; Nalitov, A. V.; Klaas, M.; Dietrich, C. P.; Kavokin, A. V.; Höfling, S.; Schneider, C., Room-temperature Tamm-plasmon exciton-polaritons with a WSe2 monolayer. Nat. Commun. 2016, 7 (1), 13328.

18. Gu, J.; Chakraborty, B.; Khatoniar, M.; Menon, V. M., A room-temperature polariton light-emitting diode based on monolayer WS2. Nature Nanotech. 2019, 14 (11), 1024-1028.

19. Sun, L.; Wang, C.-Y.; Krasnok, A.; Choi, J.; Shi, J.; Gomez-Diaz, J. S.; Zepeda, A.; Gwo, S.; Shih, C.-K.; Alù, A.; Li, X., Separation of valley excitons in a MoS2 monolayer using a subwavelength asymmetric groove array. Nat. Photonics 2019, 13 (3), 180-184.

20. $\quad$ Seyler, K. L.; Rivera, P.; Yu, H.; Wilson, N. P.; Ray, E. L.; Mandrus, D. G.; Yan, J.; Yao, W.; Xu, X., Signatures of moiré-trapped valley excitons in MoSe2/WSe2 heterobilayers. Nature 2019, 567 (7746), 66-70.

21. Gottscholl, A.; Kianinia, M.; Soltamov, V.; Orlinskii, S.; Mamin, G.; Bradac, C.; Kasper, C.; Krambrock, K.; Sperlich, A.; Toth, M.; Aharonovich, I.; Dyakonov, V., Initialization and read-out of intrinsic spin defects in a van der Waals crystal at room temperature. Nature Mater. 2020, 19 (5), 540-545.

22. Chejanovsky, N.; Mukherjee, A.; Geng, J.; Chen, Y.-C.; Kim, Y.; Denisenko, A.; Finkler, A.; Taniguchi, T.; Watanabe, K.; Dasari, D. B. R.; Auburger, P.; Gali, A.; Smet, J. H.; Wrachtrup, J., Single-spin resonance in a van der Waals embedded paramagnetic defect. Nature Mater. 2021, 20 (8), 1079-1084.

23. Mizuochi, N.; Makino, T.; Kato, H.; Takeuchi, D.; Ogura, M.; Okushi, H.; Nothaft, M.; Neumann, P.; Gali, A.; Jelezko, F.; Wrachtrup, J.; Yamasaki, S., Electrically driven singlephoton source at room temperature in diamond. Nat. Photonics 2012, 6 (5), 299-303.

24. Lohrmann, A.; Iwamoto, N.; Bodrog, Z.; Castelletto, S.; Ohshima, T.; Karle, T. J.; Gali, A.; Prawer, S.; McCallum, J. C.; Johnson, B. C., Single-photon emitting diode in silicon carbide. Nat. Commun. 2015, 6, 7783.

25. Grotz, B.; Hauf, M. V.; Dankerl, M.; Naydenov, B.; Pezzagna, S.; Meijer, J.; Jelezko, F.; Wrachtrup, J.; Stutzmann, M.; Reinhard, F.; Garrido, J. A., Charge state manipulation of qubits in diamond. Nat Commun 2012, 3, 729.

26. Widmann, M.; Niethammer, M.; Fedyanin, D. Y.; Khramtsov, I. A.; Rendler, T.; Booker, I. D.; UI Hassan, J.; Morioka, N.; Chen, Y.-C.; Ivanov, I. G.; Son, N. T.; Ohshima, T.; Bockstedte, M.; Gali, A.; Bonato, C.; Lee, S.-Y.; Wrachtrup, J., Electrical Charge State Manipulation of Single Silicon Vacancies in a Silicon Carbide Quantum Optoelectronic Device. Nano Lett. 2019, 19 (10), 7173-7180.

27. Anderson, C. P.; Bourassa, A.; Miao, K. C.; Wolfowicz, G.; Mintun, P. J.; Crook, A. L.; Abe, H.; Ul Hassan, J.; Son, N. T.; Ohshima, T.; Awschalom, D. D., Electrical and optical control of single spins integrated in scalable semiconductor devices. Science 2019, 366 (6470), 1225-1230. 
28. Brenneis, A.; Gaudreau, L.; Seifert, M.; Karl, H.; Brandt, M. S.; Huebl, H.; Garrido, J. A.; KoppensFrank, H. L.; Holleitner, A. W., Ultrafast electronic readout of diamond nitrogenvacancy centres coupled to graphene. Nature Nanotech. 2015, 10 (2), 135-139.

29. Cano, D.; Ferrier, A.; Soundarapandian, K.; Reserbat-Plantey, A.; Scarafagio, M.; Tallaire, A.; Seyeux, A.; Marcus, P.; Riedmatten, H. d.; Goldner, P.; Koppens, F. H. L.; Tielrooij, K.-J., Fast electrical modulation of strong near-field interactions between erbium emitters and graphene. Nat. Commun. 2020, 11 (1), 4094.

30. Doi, Y.; Makino, T.; Kato, H.; Takeuchi, D.; Ogura, M.; Okushi, H.; Morishita, H.; Tashima, T.; Miwa, S.; Yamasaki, S.; Neumann, P.; Wrachtrup, J.; Suzuki, Y.; Mizuochi, N., Deterministic Electrical Charge-State Initialization of Single Nitrogen-Vacancy Center in Diamond. Physical Review X 2014, 4 (1), 011057.

31. Auburger, P.; Gali, A., Towards ab initio identification of paramagnetic substitutional carbon defects in hexagonal boron nitride acting as quantum bits. Phys. Rev. B 2021, 104 (7), 075410.

32. Jara, C.; Rauch, T.; Botti, S.; Marques, M. A. L.; Norambuena, A.; Coto, R.; Castellanos-Águila, J. E.; Maze, J. R.; Munoz, F., First-Principles Identification of Single Photon Emitters Based on Carbon Clusters in Hexagonal Boron Nitride. The Journal of Physical Chemistry A 2021, 125 (6), 1325-1335. 